\title{
Selecting the optimal image segmentation strategy in the era of multitracer multimodality imaging: a critical step for image-guided radiation therapy
}

\author{
Sandip Basu
}

Received: 18 November 2008 / Accepted: 25 November 2008/Published online: 8 January 2009

(C) Springer-Verlag 2008

The study by Vees et al. [1] illustrates the potential application of the image segmentation approach in a promising "non-FDG" PET tracer that is being investigated for its potential in patients with high-grade glioma. This study is a comparative evaluation of the various image segmentation techniques for the delineation of gross tumour volume (GTV) in this group of patients. The study illustrates the complexity of current image segmentation algorithms and vividly demonstrates the difference in the results obtained and the possible implications for brain tumour GTV delineation, and in so doing sounds a cautionary note. The authors deserve recognition for employing this novel quantitative approach with novel PET tracers in this promising arena of image-guided radiotherapy.

A number of innovative PET tracers are being tested around the world for probing biochemical and pharmacological functions, and combined with a further understanding of human biology at the molecular level, the area of imageguided radiation therapy continues to evolve. The availability of PET-CT as a practical tool has further enhanced the role of metabolic imaging in radiotherapy planning in several malignancies. ${ }^{18}$ F-Fluoroethyl-L-tyrosine (FET) is a promising ${ }^{18} \mathrm{~F}$-labelled amino acid analogue that has been shown to reliably differentiate tumour recurrence from reactive changes following various therapies, particularly external radiation therapy $[2,3]$. This tumour specificity has been the major reason for the interest generated in this tracer, particularly in the setting of cerebral glioma. Another

This Editorial commentary refers to the article http://dx.doi.org/ 10.1007/s00259-008-0943-6

\section{S. Basu $(\bowtie)$}

Radiation Medicine Centre (BARC),

Tata Memorial Hospital Annexe,

Parel,

Bombay 400012, India

e-mail: drsanb@yahoo.com potential area of interest is its usefulness in assessing tumour grading where standard evaluations have been considered of little value because of marked overlap reported between histological grades $[4,5]$. A recent report on FET uptake kinetics in untreated glioma patients has demonstrated a significant difference in the uptake values in the early phase (0-10 min after injection) but not in the later period (30$40 \mathrm{~min}$ after injection) between low- and high-grade gliomas [4]. On the basis of their results, the authors hypothesized that differentiation between low- and high-grade gliomas is possible by taking into account the different kinetic behaviour of FET in these tumours. The preliminary results demonstrate that high- and low-grade brain tumours exhibit different uptake kinetics of FET, and a kinetic analysis of FET PET, therefore, may provide important information on the differentiation of suspected brain lesions [4]. The factors that have been suggested to contribute to the different kinetic behaviour in low- and high-grade tumours include increased angiogenesis and intratumoral microvessel density and increased amino acid transporter expression in tumour vessels $[4,5]$.

In recent years, multimodality imaging information is being increasingly examined for its potential utility in radiotherapy treatment planning for cancer patients. It is imperative to utilize and integrate all the imaging data available for target image coregistration and segmentation to generate combined information to define the overall biophysical characteristics of the tumour. Methods for accurate tumour volume segmentation of PET images have been under investigation in recent years partly as a result of the increased use of PET in radiation treatment planning. At this point, however, image segmentation for PET target volume delineation in radiation treatment planning is "a work in progress". Also, concurrent multimodality segmentation methods have been proposed as feasible and accurate for integrating multimodality imaging information (consisting of 
combinations of coregistered PET, CT, or MR datasets) that can be potentially useful for radiotherapy treatment planning for cancer patients [6].

In the present study, the accuracy of different segmentation strategies presently available was compared in a prospective setting. The authors concluded that the selection of the most appropriate FET PET-based segmentation algorithm is crucial for correct delineation of the resulting GTV. The study results highlight the limitations associated with some of the segmentation algorithms (e.g. 2.5 standardized uptake values cut-off and the gradient finding GTV approaches) compared to the adaptive thresholding technique based on the signal-to-background ratio (SBR) and its impact on radiotherapy planning in patients with cerebral glioma. The study is important from several standpoints. Firstly, it employed novel quantitative approaches such as PET image segmentation with non-FDG PET tracers (in this case ${ }^{18} \mathrm{~F}$-FET) for planning radiotherapy in brain tumours where it can have significant implications for patient management. Secondly, it was a comparative evaluation study of different image segmentation approaches. It is possible that with the emergence of more specific PET tracers, the conventional segmentation approaches that appear optimal with FDG might not produce desirable results with others, as observed by these investigators. Hence it is critical to examine head-to-head the results obtained with these segmentation algorithms for these promising novel PET tracers that are likely to be utilized to delineate the tumour volume in the future. Although there has been significant improvement in the area of quantitative PET over the last few years, such studies continue to be in their infancy.

The results of the present study can be extrapolated to other malignancies where FET PET has shown a higher specificity with reasonable sensitivity as well $[7,8]$. This may ultimately result in enhanced dose conformance to the target, with significant implications for patients being considered for radiotherapy. It is imperative that the application of these findings help define viable tumour boundaries with better precision, aid the progress of image segmentation, and assist in understanding their importance in accurately characterizing active tumour volume.

\section{References}

1. Vees H, Senthamizhchelvan S, Miralbell R, Weber DC, Ratib O, Zaidi H. Assessment of various strategies for (18)F-FET PET-guided delineation of target volumes in high-grade glioma patients. Eur J Nucl Med Mol Imaging 2008. doi:10.1007/s00259-008-0943-6.

2. Pöpperl G, Goldbrunner R, Gildehaus FJ, Kreth FW, Tanner P, Holtmannspötter M, et al. O- $\left(2-\left[{ }^{18} \mathrm{~F}\right]\right.$ fluoroethyl)-L-tyrosine PET for monitoring the effects of convection-enhanced delivery of paclitaxel in patients with recurrent glioblastoma. Eur J Nucl Med Mol Imaging 2005;32:1018-25. doi:10.1007/s00259-005-1819-7.

3. Popperl G, Gotz C, Rachinger W, Gildehaus FJ, Tonn JC, Tatsch K. Value of O-(2-[ $\left.{ }^{18} \mathrm{~F}\right]$ fluoroethyl)-L-tyrosine PET for the diagnosis of recurrent glioma. Eur J Nucl Med Mol Imaging 2004;31:1464-70. doi:10.1007/s00259-004-1590-1.

4. Weckesser M, Langen KJ, Rickert CH, Kloska S, Straeter R, Hamacher K, et al. O-(2-[(18)F]fluoroethyl)-L-tyrosine PET in the clinical evaluation of primary brain tumours. Eur J Nucl Med Mol Imaging 2005;32:422-9. doi:10.1007/s00259-004-1705-8.

5. Miyagawa T, Oku T, Uehara H, Desai R, Beattie B, Tjuvajev J, et al. "Facilitated" amino acid transport is upregulated in brain tumors. J Cereb Blood Flow Metab 1998;18:500-9. doi:10.1097/ 00004647-199805000-00005.

6. El Naqa I, Yang D, Apte A, Khullar D, Mutic S, Zheng J, et al. Concurrent multimodality image segmentation by active contours for radiotherapy treatment planning. Med Phys 2007;34:4738-49. doi:10.1118/1.2799886.

7. Pauleit D, Zimmermann A, Stoffels G, Bauer D, Risse J, Flüss MO, et al. 18F-FET PET compared with 18F-FDG PET and CT in patients with head and neck cancer. J Nucl Med 2006;47:256-61.

8. Balogova S, Périé S, Kerrou K, Grahek D, Montravers F, Angelard $\mathrm{B}$, et al. Prospective comparison of FDG and FET PET/CT in patients with head and neck squamous cell carcinoma. Mol Imaging Biol 2008;10:364-73. doi:10.1007/s11307-008-0155-2. 